\title{
A Versatile "Click" Chemistry Precursor of Functional Polystyrene Nanoparticles
}

By Lorea Oria, Roberto Aguado, José A. Pomposo* and Juan Colmenero

[*] Prof. J. A. Pomposo

Donostia International Physics Center, Paseo Manuel de Lardizabal 4, 20018 San Sebastián, (Spain)

E-mail: josetxo_pomposo@ehu.es

Prof. J. Colmenero

Donostia International Physics Center, Paseo Manuel de Lardizabal 4, 20018 San Sebastián, (Spain)

Centro de Física de Materiales (CSIC, UPV/EHU)-Materials Physics Center, Apartado 1072, 20080 San Sebastián (Spain)

Departamento de Física de Materiales, Universidad del País Vasco (UPV/EHU), Apartado 1072, 20080 San Sebastián (Spain)

Prof. R. Aguado, L. Oria

Facultad de Ciencia y Tecnología, Universidad del País Vasco (UPV/EHU), 48940 Lejona (Spain)

Keywords: Nanoparticles, Polymeric Materials, Hierarchical Structures

Functional nanoparticles (NPs) are becoming important building blocks in the emerging fields of nanomedicine, nanolithography and nanoelectronics, among others. ${ }^{[1]}$ Several unexpected nanoscale effects have been discovered for these new materials in recent years. ${ }^{[2-4]}$ Useful synthetic routes to single-molecule polymeric NPs in the $2-15 \mathrm{~nm}$ size range with welldefined arrangement of functional groups are certainly scarce in spite of the enormous potential predicted for all-polymer nanocomposites. ${ }^{[5]}$ Intramolecular cross-linking under appropriate (e.g. diluted) reaction conditions of individual macromolecules containing complementary cross-linkable groups along the polymer chain is one of these approaches. ${ }^{[6-}$ ${ }^{12]}$ A second one relies on the use of homobifunctional cross-linkers and reaction conditions promoting single-chain collapse of macromolecules containing appropriate reactive groups 
along the chain. ${ }^{[13-15]}$ The main requirements for a versatile synthetic route to single-chain cross-linked polymeric NPs are: ${ }^{[6]}$ i) easy incorporation of coupling precursors into the individual polymer chains, ii) highly efficient and selective cross-linking (coupling) reaction, and iii) appropriate room temperature reaction conditions favouring intramolecular coupling versus intermolecular cross-linking.

In recent years, exquisite control over the composition, molecular weight and molecular weight distribution of synthetic macromolecules (including block and random copolymers) has been achieved using (user-friendly) reversible-deactivation radical polymerization (RDRP) techniques ${ }^{[16]}$ such as nitroxide-mediated polymerization (NMP), atom transfer radical polymerization (ATRP) or reversible addition-fragmentation chain transfer (RAFT) polymerization. Additionally, "click" chemistry methodologies are currently being used in polymer and materials science for easy and almost quantitative functionalization of synthetic polymers, biomolecules and inorganic nanoparticles. ${ }^{[17]}$ "Click" chemistry, ${ }^{[18]}$ and specifically the $\mathrm{Cu}^{\mathrm{I}}$-catalyzed [3+2] cycloaddition of alkynes and azides, ${ }^{[19,20]}$ is a selective and very efficient kind of reactions that can be performed under extremely mild conditions with high yields, good functional group tolerance and essentially no by-products.

In fact, the combination of RDRP and "click" chemistry techniques meet all the requirements for the synthesis of well-defined single-chain cross-linked polymeric NPs. ${ }^{[6]}$ Hence, individual poly(methyl methacrylate) (PMMA) NPs were synthesized by RAFT terpolymerization of methyl methacrylate and alkyne- and azide- containing methacrylate monomers followed by r.t. intramolecular "click" coupling under diluted conditions using a continuous addition technique. By tailoring the amount of azide groups on the surface of the PMMA-NPs, a second "click" reaction was allowed opening the way to extremely small bioconjugated NPs (e.g. amino acid decorated PMMA-NPs). Nevertheless, random (statistical) terpolymerization is very difficult to perform in a controlled manner for monomers having very different polymerization rates and monomer reactivity ratios.

Here we report a new and versatile "click" chemistry precursor of functional polystyrene (PS) NPs prepared by the use of both RAFT copolymerization and a subsequent very efficient functionalization step. This procedure reduces the need for employing the random terpolymerization reaction, being the "clickable" terpolymer formed from a convenient copolymer precursor. Additionally, the resulting functional NPs are excellent raw materials for the synthesis of other complex, soft nano-objects. Specifically, we have investigated the kinetics of RAFT copolymerization of 4-chloromethyl styrene, CMS, and an alkyne-containing methacrylate monomer (2-methyl-acrylic acid 3-trimethylsilanyl-prop-2- 
ynyl ester, TSPMA) to obtain statistical copolymers. Reactivity ratios obtained for the above styrenic and methacrylic monomers were 1.1 and 1.0 by the Fineman-Ross method, ${ }^{[21]}$ and 1.3 and 0.9 by the Kelen-Tüdös method, ${ }^{[22]}$ certifying the statistical (random) nature of the resulting copolymers (see Supporting Information). The importance of the controlled copolymer microstructure on the morphology of the resulting NPs has been highlighted by Monte Carlo simulations. Random copolymers displayed the expected spherical, core-shell morphology after intramolecular collapse whereas elongated, multiple core structures were observed for uniform (alternating) copolymers. ${ }^{[23]}$

Good control over the RAFT copolymerization process leading to well-defined poly(4chloromethyl styrene $\mathrm{x}_{\mathrm{x}}$-co-2-methyl-acrylic acid 3-trimethylsilanyl-prop-2-ynyl ester ${ }_{\mathrm{y}}$ ) copolymers, 1, was supported by: i) observed linear semilogarithmic kinetic plots, ${ }^{[24]} \ln \left(\left[\mathrm{M}_{0}\right]\right.$ / $[\mathrm{M}])$ vs $\mathrm{t}$, where $\left[\mathrm{M}_{0}\right]$ and $[\mathrm{M}]$ represent the initial monomer concentration and the monomer concentration at time $t$, respectively, ii) linear increase of the number-average molecular weight $\left(\mathrm{M}_{\mathrm{n}}\right)$ with conversion (see Figure 1a) and, iii) very narrow polydispersity index (PDI $\equiv \mathrm{M}_{\mathrm{w}} / \mathrm{M}_{\mathrm{n}}$ ), typically between 1.09 and 1.24 .

It is worth mentioning the very good agreement between the value of the kinetic propagation constant $\left(\mathrm{k}_{\mathrm{p}}\right)$ obtained by matching the evolution of the conversion, $\mathrm{M}_{\mathrm{n}}$ and PDI with time to the corresponding RAFT model ${ }^{[25]}$ (see Supporting Information) and those obtained from both Fineman-Ross ${ }^{[21]}$ and Kelen-Tüdös ${ }^{[22]}$ models $\left(\mathrm{k}_{\mathrm{p}}=2.2 \times 10^{6} 1 \mathrm{~mol}^{-1} \mathrm{~h}^{-1}\right.$ and $\mathrm{k}_{\mathrm{p}}=2.4 \times 10^{6} 1 \mathrm{~mol}^{-1} \mathrm{~h}^{-1}$, respectively). The appropriate modelling of the random RAFT copolymerization process was further confirmed by synthesizing different poly(4chloromethyl styrene $\mathrm{x}_{\mathrm{x}}$-co-2-methyl-acrylic acid 3-trimethylsilanyl-prop-2-ynyl ester ${ }_{\mathrm{y}}$ ) copolymers, 1 , (from $x=0.95$ to $x=0.05$ ) with pre-defined characteristics. A good example is copolymer 2 with 4-chloromethyl styrene molar fraction of $\mathrm{x} \sim 0.8, \mathrm{M}_{\mathrm{n}}=31.300 \mathrm{~g} \mathrm{~mol}^{-1}$ and PDI $=1.1$ that can be used after appropriate functionalization as a versatile "click" chemistry precursor of well-defined chlorinated PS-NPs. Hence, partial replacement of the chlorine groups by azide moieties in the copolymer by treatment with sodium azide in DMF (see Figure 1b and 1c) led to terpolymer $\mathbf{3}$ containing almost equimolar quantities of azide (0.19) and alkyne (0.21) functionalites that was able to undergo intramolecular "click" coupling at room temperature under appropriated conditions (Figure 1d). ${ }^{1} \mathrm{H}$ NMR spectroscopy was employed to follow the kinetics for the azidation reaction of $\mathbf{2}$ (Figure $\mathbf{1 b}$ ). The corresponding kinetic constant was found to be $0.0951 \mathrm{~mol}^{-1} \mathrm{~h}^{-1}$ and the reaction proceeded with excellent reaction yield ( $92 \%$, Figure 1c). The quantitative disappearance of the azide vibration band in terpolymer 3 upon 1,2,3-triazole formation was confirmed by 
both ${ }^{1} \mathrm{H}$ NMR spectroscopy and Fourier transform infra-red (FTIR) spectroscopy (see Supporting Information).

The resulting NPs can be dispersed in several solvents, such as toluene, tetrahydrofurane (THF) or dimethyl formamide (DMF). A nanoparticle diameter of $4.2 \pm 0.9$ $\mathrm{nm}$ was determined by dynamic light scattering (DLS) experiments in THF. The theoretical size of a poly(4-chloromethyl styrene) chain when fully compacted to the density of a solid spherical nanoparticle, can be estimated by using the following equation:

$$
D_{p}=\left(\frac{6 M_{n}}{\pi \rho N_{A}}\right)^{1 / 3}
$$

where $D_{p}$ is the diameter of the nanoparticle, $M_{n}$ is the number-average molecular weight of the polymer, $\rho$ is the density and $N_{A}$ is Avogadro's number. By using $M_{n}=31.300 \mathrm{~g} \mathrm{~mol}^{-1}$ and $\rho=1.1 \mathrm{~g} \mathrm{~cm}^{-3}$, we obtain $D_{p}=4.5 \mathrm{~nm}$ in excellent agreement with the DLS experimental data. An illustration of the morphology of the Cl-PS-NPs as observed by atomic force microscopy (AFM) is shown in Figure 2a. It is worth noticing that due to the high dilution employed to avoid NP agglomeration, only a few NPs are indeed observed in Figure 2a. As expected for the statistical distribution of reacting groups in the precursor, the NPs display a spherical morphology with a particle size in the dried state of about $2.5 \mathrm{~nm}$. The difference in size obtained from DLS and AFM measurements could be attributed to partial swelling of the nanoparticles ${ }^{[26]}$ in solution and the slight spreading of the NPs over the substrate upon drying, ${ }^{[27]}$ respectively.

Since the PS-NPs retain the Cl- functionality after their synthesis, they offer the advantages of such chemistry and the inherent characteristics of polymeric NPs. Interestingly, the synthesized ultra-small Cl-PS-NPs display good thermal stability up to $\sim 200{ }^{\circ} \mathrm{C}$ (Figure 2b). Melt viscosity reduction in conventional thermoplastic materials due to polymeric NP addition has been reported by Mackay et al. ${ }^{[28]}$ To the best of our knowledge, no similar finding has been made in rubber nanocomposites even when viscosity reduction during rubber injection moulding of products with thin cross sections is a hot demand in industry. We have prepared nanocomposites of neat natural rubber (cis-1,4-polyisoprene) and ultra-small functional NPs by a rapid precipitation method using toluene as solvent and cold methanol as precipitant. The resulting nanocomposites were dried under vacuum at $100{ }^{\circ} \mathrm{C}$ until constant weight, prior to melt viscosity measurements at that temperature (Figure 2c). Careful TGA experiments were performed to confirm that solvent effects were completely absent in the dried samples. As illustrated in Figure 2c, a significant viscosity drop was found as a 
consequence of the presence of the ultra-small nanoparticles. To the best of our knowledge, this is the first report of rubber nanocomposites with reduced melt viscosity arising from the presence of well-dispersed individual polymeric NPs. Moreover, only a small weight percentage of nanoparticles was required to significantly decrease the viscosity of the neat rubber matrix. The involved nanoscale mechanism by which nanoparticles decrease viscosity is not well-understood at present. In addition, further experiments are required to investigate the rheology behaviour of rubber nanocomposite formulations containing carbon black, vulcanization accelerators, antioxidants and other additives, which are certainly out of the scope of the present paper.

It is worth noticing that the $\mathrm{Cl}$ - functional groups allow nanoparticle surface decoration (see Figure 2d) using atom transfer radical polymerization (ATRP) strategies (i.e. by employing the nanoparticles as multifunctional initiators). ${ }^{[29]}$ Controlled azide functionalization of the remaining $\mathrm{Cl}$ - groups opens the way to the synthesis of new bioconjugated hybrids such as NP-oligopeptide, NP-protein, NP-oligonucleotide and NPcarbohydrate bioconjugates. ${ }^{[30,31]}$ Improved Förster resonance energy transfer (FRET) could be also envisaged following recent strategies. ${ }^{[32]}$ As a related example, we illustrate in Figure 2e the fluorescence spectrum of NPs in which coupling of triazole-benzene-triazole units has taken place by using an appropriate bifunctional cross-linker (1,4-diethynylbenzene). After excitation of the NPs using a wavelength of $350 \mathrm{~nm}$, a fluorescence emission band was observed with two peaks located at about 391 and $407 \mathrm{~nm}$, and a small shoulder at $424 \mathrm{~nm}$. Control experiments in which triazole-benzene-triazole conjugation was perturbed or was absent failed to show the improved fluorescence behaviour displayed in Figure 2e. An indeep study of the interesting possibilities offered by these functional NPs for the above mentioned applications will be the subject of a separate publication.

In summary, a versatile "click" chemistry precursor has been synthesized using a reversible-deactivation radical polymerization technique and the efficient r.t. preparation of ultra-small functional polystyrene nanoparticles thereof has been demonstrated. As a result of the multifunctional nature of these materials, improved rheology behaviour (when mixed with a elastomeric polymer) and useful fluorescence emission (when aromatic conjugation is maximized) has been demonstrated. The resulting well-defined nanoparticles are expected to become raw materials for the further synthesis of other complex, soft nano-objects bridging the gap between synthetic and natural polymers. ${ }^{[33]}$ 


\section{Experimental}

Materials: Unless otherwise stated, all the chemicals used in the syntheses were purchased from Sigma-Aldrich (reagent grade) and used without further purification. Silica gel for flash chromatography was Merck - Kieselgel 60 (0.040-0.063 mm) and aluminium oxide was Fluka-Brockmann Activity I. 2-Phenyl-2-yl dithiobenzoate (cumyl dithiobenzoate, CDB) [34] and 2-methyl-acrylic acid 3-trimethylsilanyl-prop-2-ynyl ester (TSPMA) [35] where synthesized and purified as reported in the literature.

Preparation of poly(4-chloromethyl styrene $x_{-}$-co-2-methyl-acrylic acid 3-trimethylsilanylprop-2-ynyl ester ${ }_{y}$ ) copolymers, 1: Different RAFT copolymerization reactions of 4chloromethyl styrene (CMS) and TSPMA were investigated by modifying the $[\mathrm{CMS}] /[\mathrm{TSPMA}]$ and $[\mathrm{CDB}] /[\mathrm{AIBN}]$ ratios and the reaction temperature, both in solution (toluene as solvent) and in the bulk. In a typical bulk procedure, a mixture of CMS $(5.87 \mathrm{~g}$, $38.46 \mathrm{mmol})$, TSPMA (1.29 g, $11 \mathrm{mmol})$, CDB (0.019 g, $0.075 \mathrm{mmol})$ and AIBN $(0.0025 \mathrm{~g}$, $0.015 \mathrm{mmol}$ ) was placed in a Schlenk flask and degassed by means of 5 freeze/pump/thaw cycles. The reaction mixture was then placed in a preheated $\left(75^{\circ} \mathrm{C}\right)$ oil bath and stirred for 24 $\mathrm{h}$ at the same temperature. The flask was then allowed to cool to room temperature and the resulting mixture was diluted with toluene $(1 \mathrm{ml})$ and precipitated into methanol. The product, 2, was filtered and dried under vacuum, to give a slightly pink solid (5.44 g, yield: $76 \%)$ that was characterized by FTIR (Nicolet Avatar 360). The composition of the copolymer was determined by ${ }^{1} \mathrm{H}$ NMR spectroscopy (Bruker UltraShield, $500 \mathrm{MHz}$ ). The mole fraction of CMS in the copolymer was found to be 0.79 . The number-averaged molecular weight and polydispersity index were $31.310 \mathrm{~g} \mathrm{~mol}^{-1}$ and 1.1 , respectively, as determined by SEC measurements in a Shimadzu SCL-10 apparatus using THF as eluent and calibrated with PS standards.

Azidation of poly(4-chloromethyl styrene $e_{x}$-co-2-methyl-acrylic acid 3-trimethylsilanyl-prop-2ynyl ester $)_{y}$ copolymers: In a typical procedure, a solution of 2 ( $1 \mathrm{~g}$. in $15 \mathrm{ml}$ of DMF) was placed in a Schlenk flask and degassed by means of 5 freeze/pump/thaw cycles. To this solution, sodium azide $(0.68 \mathrm{~g}, 10.5 \mathrm{mmol})$ was added under a nitrogen atmosphere. The reaction mixture was then placed in a preheated $\left(40{ }^{\circ} \mathrm{C}\right)$ oil bath and stirred for $24 \mathrm{~h}$ at the same temperature. A clear solution of terpolymer $\mathbf{3}$ in DMF was recovered by filtration under vacuum. After precipitation in methanol and drying, terpolymer 3 was characterized by SEC, ${ }^{1} \mathrm{H}$ NMR and FTIR.

Procedure for intramolecular "click" cycloaddition: In a typical reaction, $\mathrm{CuBr}(0,4 \mathrm{~g}, 2.79$ mmol), 2,2`-bipyridil (BIPY) (0.4 g, $2.56 \mathrm{mmol})$ and tetrabutylammonium fluoride (TBAF) (3 
$\mathrm{mL}, 0.6 \mathrm{mmol}, 0.2 \mathrm{M}$ in DMF) were dissolved under $\mathrm{N}_{2}$ in previous degassed DMF (150 mL) at $25^{\circ} \mathrm{C}$. Over this solution, a solution of $\mathbf{3}(200 \mathrm{mg}$ in $10 \mathrm{~mL}$ of DMF) was added via syringe pump (LHS 300/600 Brand $\mathrm{GmbH}$ ) at a rate of $2 \mathrm{ml} \mathrm{h}^{-1}$ under $\mathrm{N}_{2}$. After addition was completed, the solution was stirred at such temperature for $24 \mathrm{~h}$. The solution was concentrated via rota-evaporation and precipitated into a methanol/water (1/1) mixture. Then, the precipitate was washed several times with a water/ammonia (1/1) mixture and after that with pure water until $\mathrm{pH} \sim 7$. After liofilization of the precipitate for $24 \mathrm{~h}$, the resulting chlorinated polystyrene nanoparticles (Cl-PS-NPs) were dried at $40^{\circ} \mathrm{C}$ for $24 \mathrm{~h}$ under vacuum. The Cl-PS-NPs were further characterized by ${ }^{1} \mathrm{H}$ NMR, FTIR, SEC, AFM (Molecular Imaging PicoLE), DLS (Beckam Coulter N5) and TGA (TA instruments Q500) techniques.

Fluorescence Measurements: Fluorescence spectra of the triazole-bencene-triazole conjugated NPs in solution were recorded on a Fluorescence Spectrometer (Perkin-Elmer LS-45). Fluorescence microscopy pictures of the dried NPs were taken on a fluorescence microscope (Leica MF).

\section{Acknowledgements}

L. O. acknowledges Cidetec-Foundation for providing partial financial support and access to its facilities during her Undergraduate Thesis Project, as well as technical assistance through the Nanotechnology Unit staff. We thank A. Lopez (Elastomeros Riojanos, S. A.) for providing the rubber sample as a gift and M. Gimeno (Trelleborg Navex, S. A.) for performing the dynamic viscosity measurements on a PPA (Alpha Technologies) Rheometer. Supporting Information is available online from Wiley InterScience or from the author.

Received: ((will be filled in by the editorial staff))

Revised: ((will be filled in by the editorial staff)) Published online: ((will be filled in by the editorial staff))

[1] A. C. Balazs, T. E. Emrick, T. O. P. Russell, Science 2006, 314, 1107.

[2] M. E. Mackay, T. T. Dao, A. Tuteja, D. L. Ho, B. V. Horn, H.-C. Kim, C. J. Hawker, Nat. Mater. 2003, 2, 762.

[3] R. S. Krishnan, M. E. Mackay, P. M. Duxbury, A. Pastor, C. J. Hawker, B. V. Horn, M. S. Wong, S. Asokan, Nano Lett. 2007, 7, 484.

[4] R. S. Krishnan, M. E. Mackay, C. J. Hawker, B.V. Horn, Langmuir 2005, 21, 5770.

[5] A. Ruiz de Luzuriaga, H. J. Grande, J. A. Pomposo, J. Chem. Phys. 2009, 130, 084905. 
[6] A. Ruiz de Luzuriaga, N. Ormategui, H. J. Grande, I. Odriozola, J. A. Pomposo, I. Loinaz, Macromol. Rapid Commun. 2008, 29, 1156.

[7] D. Mecerreyes, V. Lee, C. J. Hawker, J. L. Hedrick, A. Wursch, W. Volksen, T. Magbitang, E. Huang, R. D. Miller, Adv. Mater. 2001, 13, 204.

[8] E. Harth, B. Van Horn, V. Y. Lee, D. S. Germack, C. P. Gonzales, R. D. Miller, C. J. Hawker, J. Am. Chem. Soc. 2002, 124, 8653.

[9] J. Jiang, S. Thayumanavan, Macromolecules 2005, 38, 5886.

[10] A. E. Cherian, F. C. Sun, S. S. Sheiko, G. W. Coates, J. Am. Chem. Soc. 2007, 129, 11350.

[11] T. A. Croce, S. K. Hamilton, M. L. Chen, H. Muchalski, E. Harth, Macromolecules 2007, $40,6028$.

[12] E. J. Foster, E. B. Berda, E. W. Meijer, J. Am. Chem. Soc. 2009, 131, 6964.

[13] J. B. Beck, K. L. Killops, T. Kang, K. Sivanandan, A. Bayles, M. E. Mackay, K. L. Wooley, C. J. Hawker, Macromolecules 2009, 42, 5629.

[14] A. Ruiz de Luzuriaga, I. Perez-Baena, S. Montes, I. Loinaz, I. Odriozola, I. García, J. A. Pomposo, Macromolecular Symposia (Submitted).

[15] D. Chen, H. Peng, M. Jiang, Macromolecules 2003, 36, 2576.

[16] K. Matyjaszewski. Controlled/Living Radical Polymerizations: From Synthesis to Materials. ACS, Washintong, DC: Oxford University Press, 2006.

[17] W. H. Binder, R. Sachsenhofer, Macromol. Rapid Commun. 2007, $28,15$.

[18] H. C. Kolb, M. G. Finn, K. B. Sharpless, Angew. Chem. Int. Ed. 2001, 40, 2004.

[19] V. V. Rostovtsev, L. G. Green, V. V. Fokin, K. B. Sharpless, Angew. Chem. Int. Edn. 2002, 41, 2596.

[20] C. W. Tornoe, C. Christensen, M. Meldal, J. Org. Chem. 2002, 67, 3057.

[21] M. Fineman, S. D. Ross, J. Polym. Sci., 1944, 66, 1594.

[22] T. Kelen, F. Tüdös, J. Macromol. Sci., Chem., 1975, A9, 1.

[23] A. Kumar, H. Nanvati, G. Kumaraswamy, J. Chem. Phys. 2007, 127, 234901.

[24] W. A. Braunecker, K. Matyjaszewski, Prog. Polym. Sci. 2007, 32, 93.

[25] Handbook of RAFT Polymerization. C. Barner-Kowollik (Ed.), Wiley-VCH, 2008.

[26] It is well known that Merrifiel resin (a copolymer of styrene and 4-chloromethyl styrene cross-linked with divinylbenzene) is swollen by THF (see, for example, A. R. Vaino, D. B. Goodin, K. D. Janda, J. Comb. Chem. 2000, 2, 330).

[27] T. E. Dukette, M. E. Mackay, B. Van Horn, K. L. Wooley, E. Drockenmuller, M. Malkoch, C. J. Hawker, Nano Lett. 2005, 5, 1704. 
[28] A. Tuteja, P. M. Duxbury, M. E. Mackay, Macromolecules 2007, 40, 9427.

[29] K. Matyjaszewski, N. V. Tsarevsky, Nature Chem. 2009, 1, 276.

[30] H. A. Klok, J. Polym. Sci. Part A: Polym. Chem. 2005, 43, 1.

[31] C. J. Hawker, K. L. Wooley, Science 2005, 309, 1200.

[32] M. D. Allen, J. Zhang, Angew. Chem. Int. Edit. 2008, 47, 500.

[33] H. G. Börner, Prog. Polym. Sci. 2009, 34, 811.

[34] PCT Int. Appl. WO 9801478 (1998), invs.: T. P. Le, G. Moad, E. Rizzardo, S. H. Thang. [35] V. Ladmiral, G. Mantovani, G. J. Clarkson, S. Cauet, J. L. Irwin, D. M. Haddleton, J. Am. Chem. Soc. 2006, 128, 4823. 
Figure 1. a) Typical linear increase of the number-average molecular weight $\left(M_{n}\right)$ with conversion during the reversible-deactivation radical copolymerization of 4-chloromethyl styrene (CMS) and 2-methyl-acrylic acid 3-trimethylsilanyl-prop-2-ynyl ester (TSPMA) monomers. b) Evolution of the $\mathrm{H}^{1}$-NMR spectra of copolymer 2 during the azidation reaction. The position of the NMR peaks associated with representative functional groups of each repeat unit involved is indicated by arrows. c) Conversion $v s$ time plot during the azidation reaction of copolymer 2. d) Synthesis of well-defined chlorinated polystyrene nanoparticles (Cl-PS-NPs) by intramolecular cycloaddition of the "click" chemistry precursor 3 at $25^{\circ} \mathrm{C}$. In the schematic picture, light circles denote 4-chloromethyl styrene units, whereas dark circles denote 1,2,3-triazole cross-links upon reaction of alkyne and azide functional groups.

\section{Fig. 1a)}

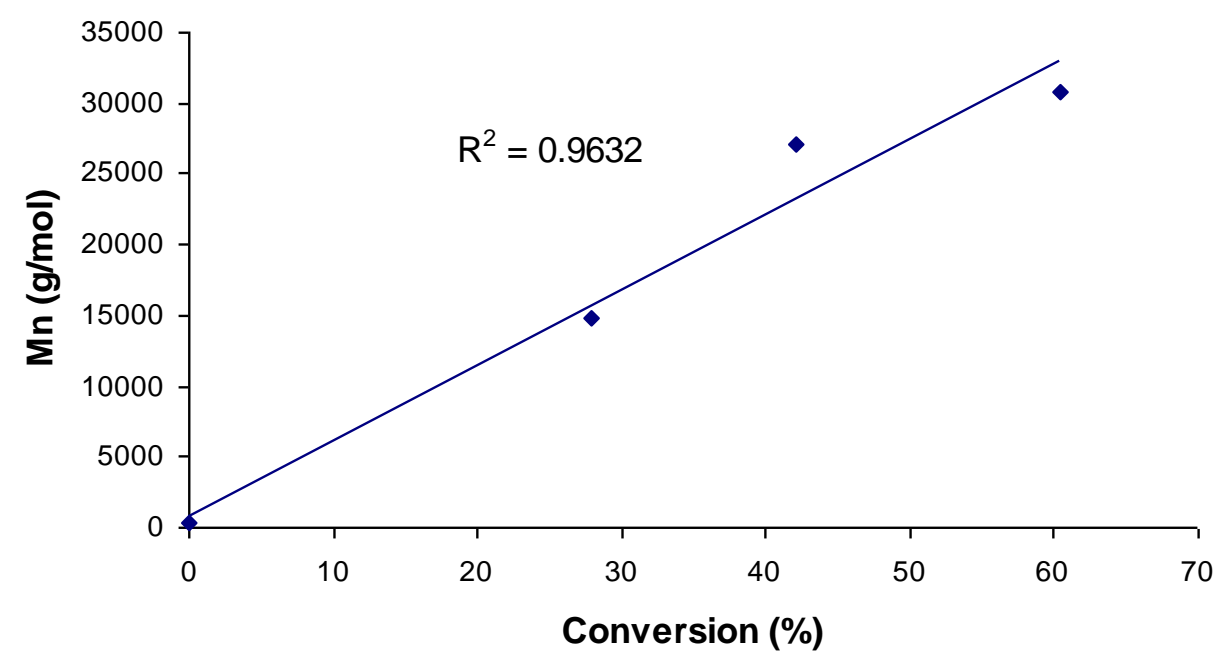


Fig. 1b)
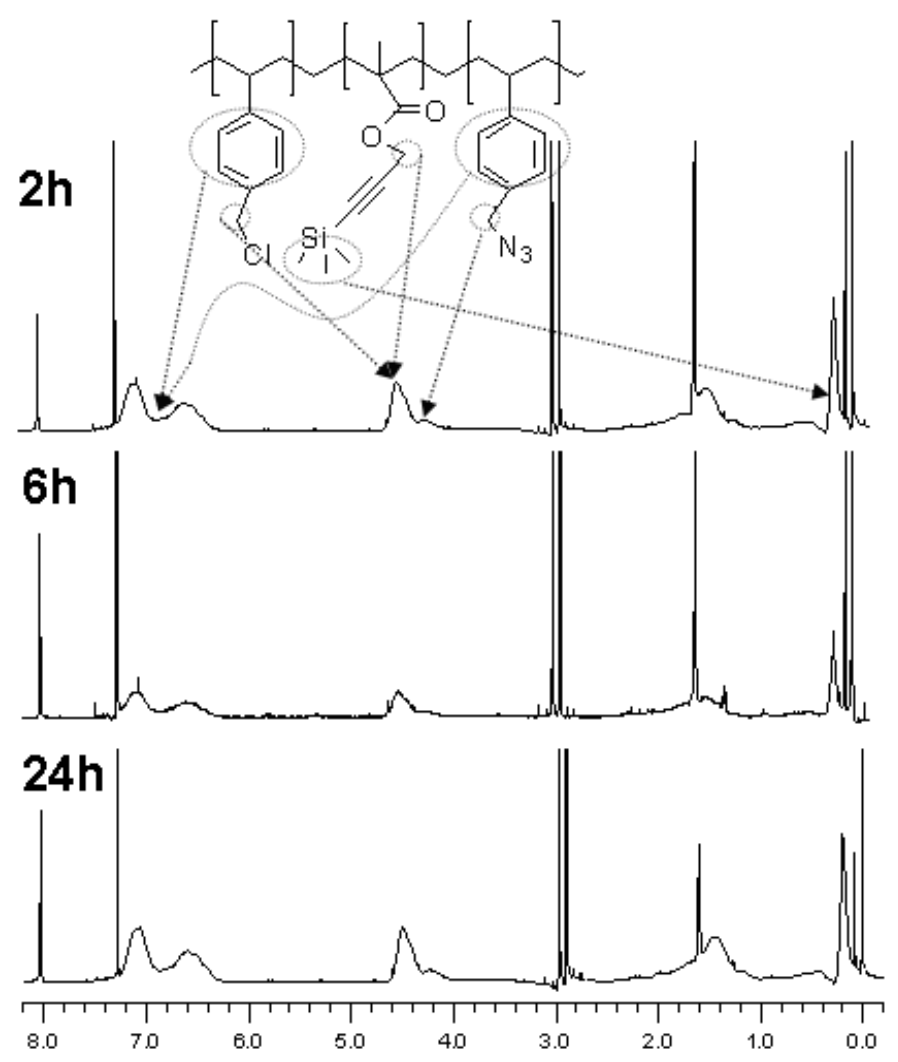

ppm 
Fig. 1c)

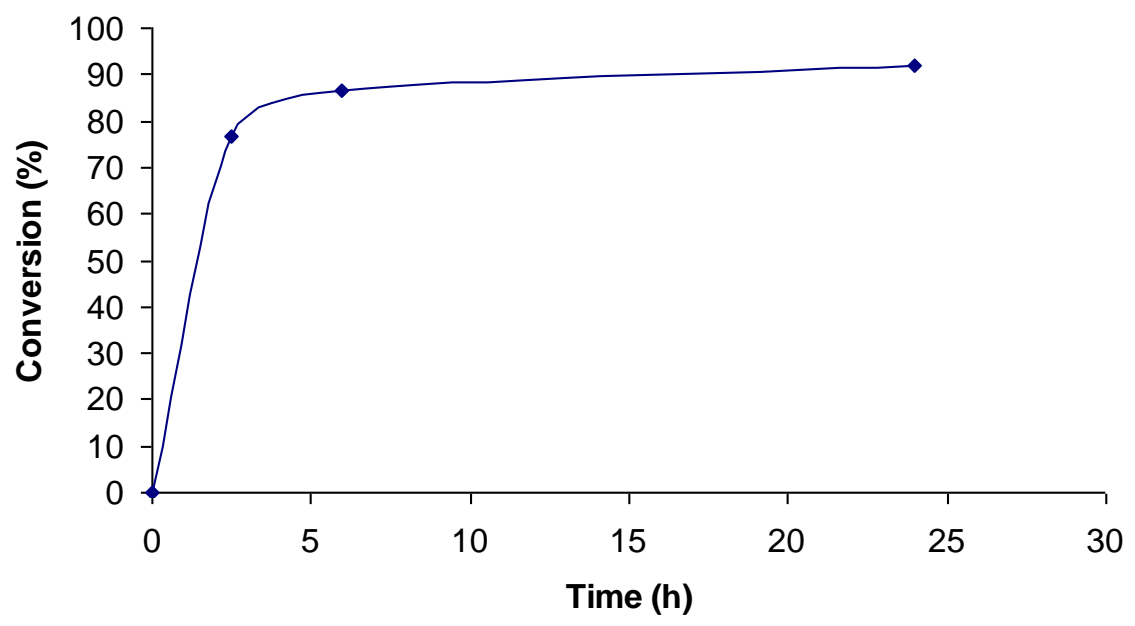


Fig. 1d)

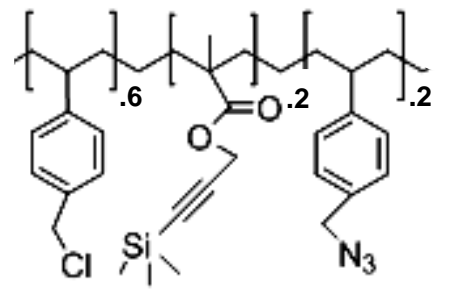

$\underset{\mathrm{CuBr}}{\stackrel{\text { TBAF,BIPY,DMF }}{\longrightarrow}}$

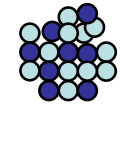

CI-PS-NP

Size $\sim 4.2 \mathrm{~nm}$

3 
Figure 2. a) Atomic force microscopy (AFM) height and phase pictures of Cl-PS-NPs and the corresponding height profile across the line drawn in left picture. b) Thermal stability of $\mathrm{Cl}$ PS-NPs as determined by thermogravimetric analysis (TGA). c) Improved rheology behaviour of natural rubber nanocomposites containing ultra-small functional PS-NPs. d) Cl-PS-NPs retain the $\mathrm{Cl}$ - functionality after their synthesis opening the way to new nanostructured multifunctional iniciators, "tadpole"-like nanoparticle-bioconjugated hybrids $(\mathrm{R}=$ oligopeptide, protein, oligonucleotide or carbohydrate) or improved Förster resonance energy transfer (FRET) nanomaterials ( $\mathrm{D}=$ donor groups, and $\mathrm{A}=$ acceptor groups). e) Fluorescence spectrum of NPs in which coupling of triazole-benzene-triazole units has taken place by using an appropriate bifunctional cross-linker (1,4-diethynylbenzene). The inset shows the raw NPs as observed by fluorescence microscopy.

Fig. 2a)
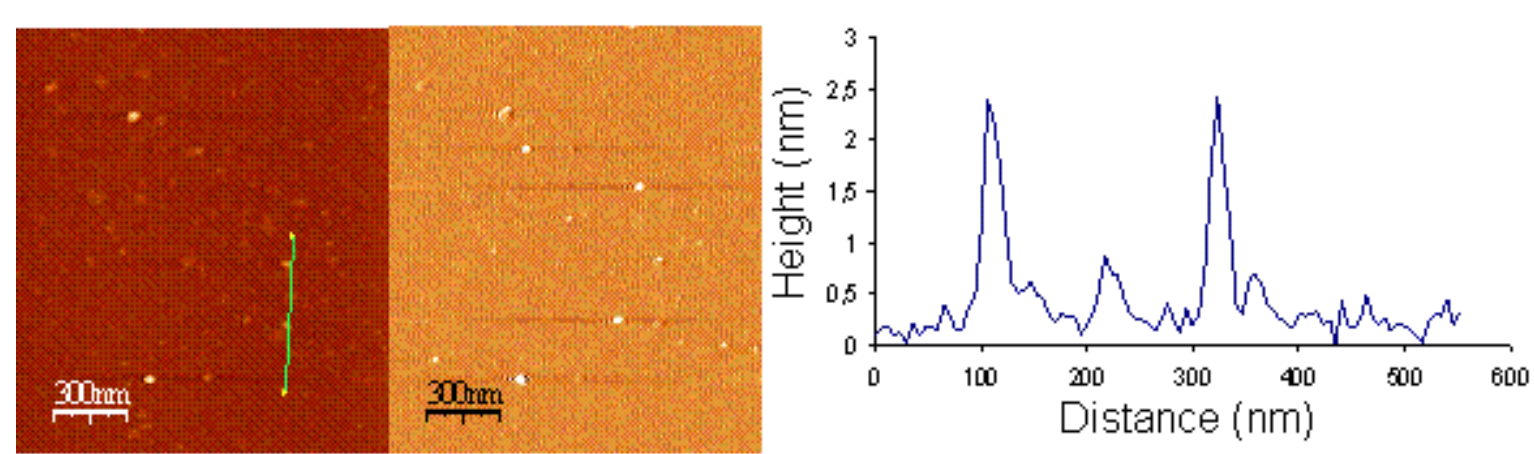
Fig. 2b)

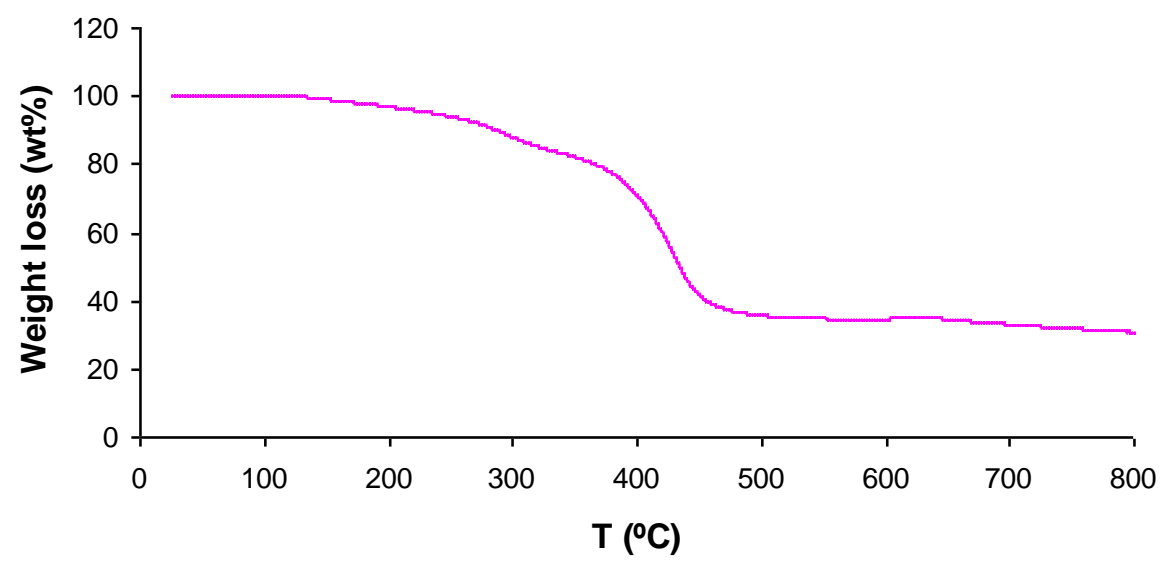


Fig. 2c)

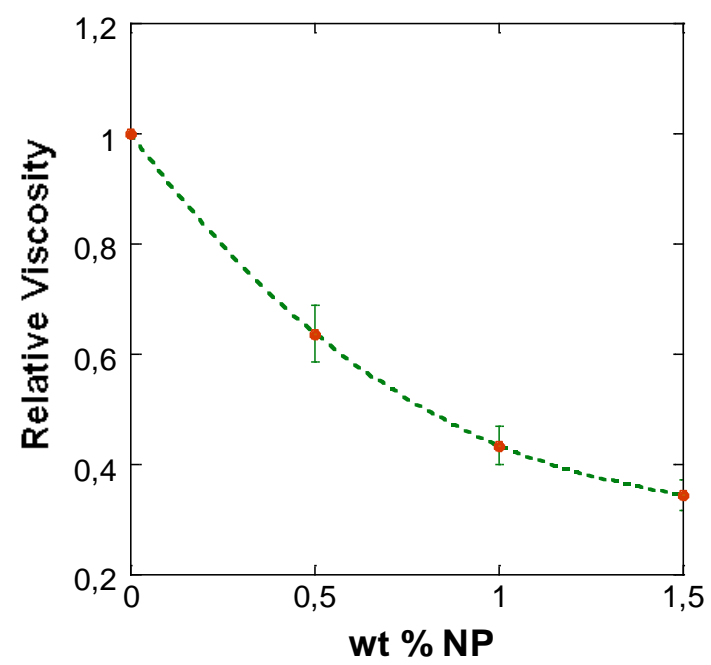


Fig. 2d)

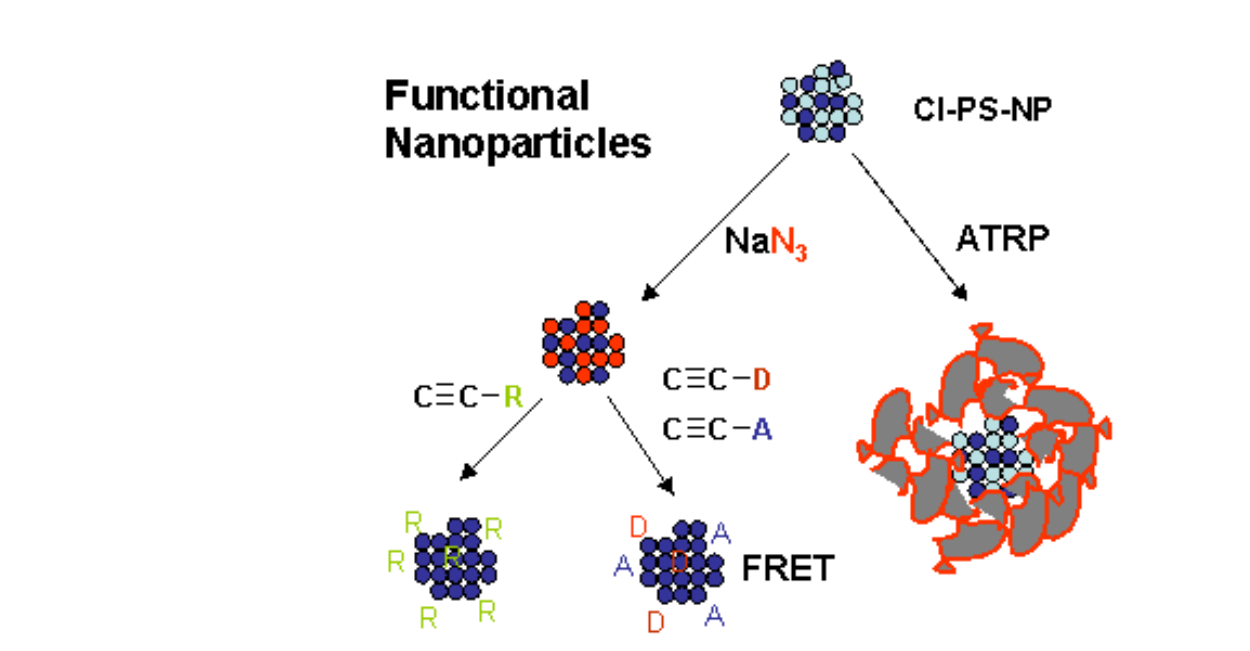


Fig. 2e)

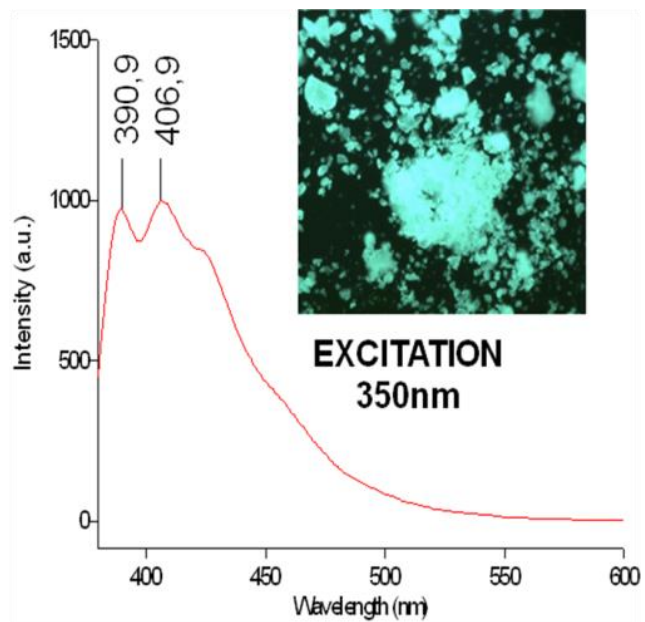

